had no effect on new total plaque formation (adjusted odds ratio-OR 0.88 [95\% $\mathrm{Cl}$ 0.64-1.21]). However, each 1-SD increase in time-averaged HDL-C associated with a $44 \%$ reduced likelihood of new non-calcified plaque formation at follow-up (adjusted OR 0.56 [95\% Cl 0.35-0.92], Figure 1). In contrast, there was no effect of time-averaged HDL-C on new mixed or calcified plaque formation. Of 98 non-calcified plaques at baseline, 42 did not change at follow-up, 32 regressed (disappeared), 16 transitioned to mixed and 8 to calcified plaques. Each SD increase in time-averaged HDL-C yielded a 2.2-fold greater likelihood of non-calcified plaque regression (adjusted OR 2.21 [95\% Cl 1.02-4.83]). Sixteen of 52 mixed plaques present at baseline transitioned to more stable calcified lesions, and time-averaged HDL-C (per 1-SD increment) predicted a 3.5-fold increased likelihood of transition of mixed to fully calcified plaque (adjusted OR 3.56 [95\% Cl 1.25-10.17]).

Conclusion: Higher HDL-C over time predicted regression of existing and decreased formation of new higher-risk non-calcified plaque. It also associated with transition of vulnerable mixed plaque to more stable fully calcified plaque. These effects were independent of RA treatment duration, prednisone dose and statin exposure.

REFERENCES:

[1] Toms TE et al. Curr Vasc Pharmacol. 2010;8:301-326.

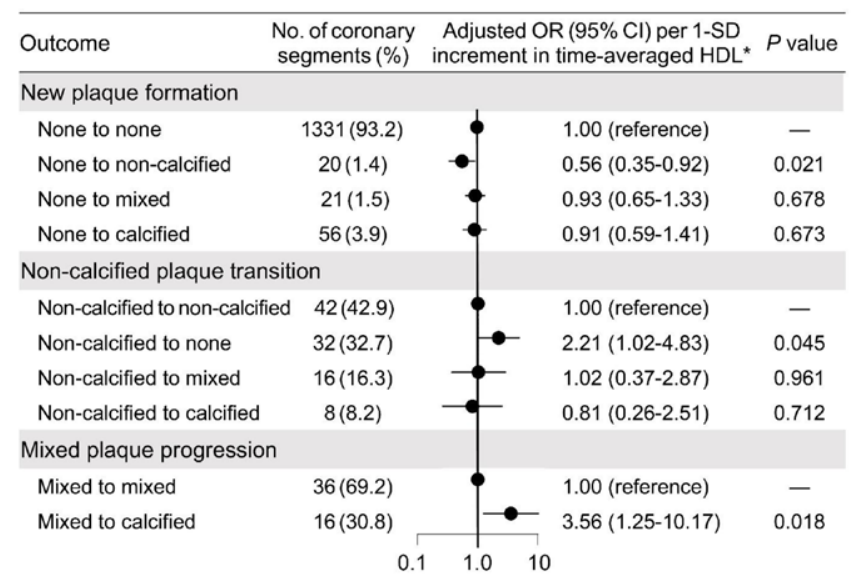

*Adjusted for Framingham D'Agostino cardiac risk score, proximal segment location, timeaveraged CRP, biologic DMARD treatment duration, statin treatment duration, cumulative prednisone dose, waist-to-height ratio, and time-averaged triglycerides.

Figure 1. Impact of HDL-C over time on coronary plaque progression in RA

Disclosure of Interests: George Karpouzas Speakers bureau: Sanofi/Genzyme/Regeneron, Consultant of: Sanofi/Genzyme/Regeneron, Grant/research support from: Pfizer, Sarah Ormseth: None declared, Elizabeth Hernandez: None declared, Matthew Budoff: None declared

DOI: 10.1136/annrheumdis-2021-eular.3383

\section{POS0217 PULSE WAVE VELOCITY ELEVATION ABOVE INDIVIDUAL REFERENCE VALUES AND AORTIC- BRACHIAL STIFFNESS MISMATCH AS EARLY MARKERS OF ARTERIAL STIFFNESS INCREASE IN PATIENTS WITH RHEUMATOID ARTHRITIS}

E. Troitskaya ${ }^{1}$, S. Velmakin ${ }^{1}$, R. Osipyants ${ }^{1}$, A. Arbuzova ${ }^{1}$, V. R. Espinoza ${ }^{2}$, Z. Kobalava ${ }^{1}$. ${ }^{1}$ Peoples' Friendship University of Russia (RUDN University), Internal diseases, Moscow, Russian Federation; ${ }^{2}$ Peoples' Friendship University of Russia (RUDN University), Int, Moscow, Russian Federation

Background: Arterial stiffness (AS) is a known predictor of cardiovascular (CV) disease. The measurement of pulse wave velocity (PWV) is considered to be a gold standard of AS assessment but the recommended threshold of $10 \mathrm{~m} / \mathrm{s}^{1}$ may not take into account multiple factors influencing PWV. Use of the proposed individual reference values may help to identify patients with AS increase despite PWV level below this threshold ${ }^{2}$. The impact of AS on CV outcomes may be mediated by the reversal of the aortic-brachial stiffness (AS gradient) ${ }^{3}$. One small study in patients with type 2 diabetes has shown that the aortic-brachial stiffness mismatch (hereafter AS mismatch) was an earlier marker of AS than PWV elevation ${ }^{4}$. Patients with rheumatoid arthritis (RA) have high $C V$ risk and may benefit from early detection of AS increase. Both approaches have not been studied in RA previously

Objectives: To evaluate the incidence of PWV elevation above individual reference values and the frequency of AS mismatch in RA

Methods: Study group included 85 patients (pts) with RA (females $77.6 \%$, aged $59.7 \pm 14.3$ years, HTN $65 \%$, mean DAS-28(CRP) $3.7 \pm 1.1$ ) and control group (40 pts matched by gender, age and risk factors). Parameters of AS were measured by applanation tonometry. Individual PWV reference values were assessed ${ }^{2}$. The AS gradient was calculated as carotid-femoral (cf)PWV/carotid-radial (cr)PWV ratio and its elevation $\geq 1$ was considered as AS mismatch. $p<0,05$ was considered significant

Results: In pts with RA with and without history of HTN mean cfPWV was $10.3 \pm 3.1$ and $7.3 \pm 1.5 \mathrm{~m} / \mathrm{s}$, respectively, mean AS gradient $-1.4 \pm 0.4$ and $1.1 \pm 0.1(p<0.001$ for trend); in controls $-9.6 \pm 1.9$ and $6.7 \pm 1.4 \mathrm{~m} / \mathrm{s}$ and $1.3 \pm 0.3$ and $0.99 \pm 0.2$, respectively ( $p<0.001$ for trend). cfPWV elevation $\geq 10 \mathrm{~m} / \mathrm{s}$ was observed in $34.1 \%$ pts with RA and $32.5 \%$ of controls: 6.7 and $6.3 \%$ of nor motensives and 49.1 and $50 \%$ of hypertensives, respectively ( $p>0.05)$. cfPWV elevation above individual reference values was observed in $41.2 \%$ RA pts and $27.5 \%$ of controls $(p=0.03)$ : in $40 \%$ and $6.3 \%$ of normotensives $(p=0.02)$ and $41.8 \%$ and $41.7 \%$ of hypertensives, respectively. After adjustment by age, gender and systolic BP cfPWV elevation above individual reference values in normotensive RA pts was independently associated with BMI (beta=0.39, $p=0.02$ ) and dyslipidemia (beta $=0.48, p=0.01$ ). The frequency of AS mismatch in RA was significantly higher compared to the controls in both normotensive and hypertensive subgroups: $76.7 \%$ vs $43.8 \%(p=0.03)$ and $94.5 \%$ vs $79.2 \% \quad(p=0.04)$ respectively. The same trend was observed in a subgroup with normal cfPWV AS mismatch was present in RA and controls in $82.1 \%$ vs $51.9 \%(p=0.004)$ in pts with PWV $\leq 10 \mathrm{~m} / \mathrm{s}$ and in $82 \%$ and $51.7 \%(p=0.04)$, respectively in pts with PWV below individual reference values.

Conclusion: Patients with RA are characterized by higher frequency of cfPWV elevation above individual reference values compared to controls irrespectively of history of HTN. This method may be more appropriate for AS evaluation than use of standard criteria in this population. AS mismatch in RA pts is highly prevalent and may be considered as an earlier marker of AS than cfPWV elevation. These findings may be used for early detection of vascular ageing in patients with RA.

\section{REFERENCES:}

[1] Williams B, et al. 2018 ESC/ESH Guidelines for the management of arterial hypertension. J Hypertens 2018;36(10):1953-2041

[2] Reference Values for Arterial Stiffness' Collaboration. Determinants of pulse wave velocity in healthy people and in the presence of cardiovas cular risk factors: 'establishing normal and reference values'. Eur Heart J. 2010;31(19):2338-50

[3] Yu S, et al. Central Versus Peripheral Artery Stiffening and Cardiovascular Risk. Arterioscler Thromb Vasc Biol. 2020;40(5):1028-1033

[4] Troitskaya, E., et al. Aortic-brachial stiffness mismatch in patients with arterial hypertension and type 2 diabetes mellitus, J Hypertens 2018;36:e191

Disclosure of Interests: None declared

DOI: 10.1136/annrheumdis-2021-eular.3889

\section{\begin{tabular}{|l|l}
\hline POS0218 TUMOR-NECROSIS FACTOR INHIBITORS \\
\hline
\end{tabular} IMPROVE AORTIC STIFFNESS IN PATIENTS WITH LONGSTANDING RHEUMATOID ARTHRITIS}

A. Giollo ${ }^{1}$, G. Cioffi ${ }^{1}$, G. Orsolini ${ }^{1}$, F. Ognibeni ${ }^{1}$, A. Dalbeni ${ }^{2}$, R. Bixio ${ }^{1}$, G. Adami ${ }^{1}$, A. Fassio ${ }^{1}$, L. Idolazzi ${ }^{1}$, D. Gatti ${ }^{1}$, M. Rossini ${ }^{1}$, O. Viapiana ${ }^{1}$ ${ }^{1}$ University of Verona, Rheumatology Section, Department of Medicine, Verona, Italy; ${ }^{2}$ University of Verona, Internal Medicine and Hypertension Unit, Department of Medicine, Verona, Italy

Background: Major cardiovascular disease (CVD) benefits of disease-modifying anti-rheumatic drugs (DMARDs) therapy occur in early RA patients with treat-totarget strategy. However, it is unknown whether long-term DMARDs treatment in established RA could be useful to improve CVD risk profile.

Objectives: The aim of this study was to comparatively describe aortic stiffness progression in patients with longstanding and established RA treated with tumor necrosis factor inhibitors (TNFi) or conventional synthetic DMARDs (csDMARDs). Methods: Ultrasound aortic stiffness index (AoSI) has to be considered a proxy outcome measure in established RA patients. We measured AoSI in a group of RA patients on long-term treatment with TNFi or csDMARDs. Eligible participants were assessed at baseline and after 12 months; changes in serum lipids, glucose and arterial blood pressure were assessed. All patients were on stable medications during the entire follow-up.

Results: We included 107 (64 TNFi and 43 csDMARDs) RA patients. Most patients (74\%) were in remission or low disease activity and had some CVD risk factors (45.8\% hypertension, $59.8 \%$ dyslipidemia, $45.3 \%$ smoking; table 1). The two groups did not differ significantly for baseline AoSI $(5.95 \pm 3.73 \%$ vs $6.08 \pm 4.20 \%$, $\mathrm{p}=0.867$ ). Follow-up AoSI was significantly increased from baseline in the csDMARDs group $(+1.00 \%$; $p<0.0001)$ but not in the TNFi group $(+0.15 \%, p=0.477)$. Patients on TNFi had significantly lower follow-up AoSI from baseline than the csDMARDs group ( $-1.02 \%, \mathrm{p}<0.001$; ANCOVA corrected for baseline AoSI, age and systolic blood pressure). Furthermore, follow-up AoSI was significantly lower in TNFi users with 1-2 or >2 CVD risk factors than in those without (figure 1). Conclusion: Long-term treatment with TNFi was associated with reduced aortic stiffness in patients with established RA and several CVD risk factors. 